\title{
SUR L'ERGODICITÉ DU FLOT GÉODÉSIQUE EN COURBURE NÉGATIVE OU NULLE
}

\author{
par Yves COUDÈNE
}

RÉSUMÉ. Cet article est consacré à la dynamique du flot géodésique sur les variétés à courbure négative ou nulle. Après avoir détaillé quelques résultats de dynamique topologique, on étudie les propriétés ergodiques du flot géodésique sur les variétés de rang un, de trois points de vue différents: d'abord relativement à la mesure riemannienne, ensuite par une approche entropique, enfin par des techniques de généricité.

\section{INTRODUCTION}

Ces notes sont issues d'un cours donné à l'université de Tours, dans le cadre du groupement de recherche Platon, en janvier 2010. Elle constituent un survol des résultats concernant l'ergodicité du flot géodésique en courbure négative ou nulle, de rang un.

Les variétés à courbure négative ou nulle sont celles qui sont les plus proches des espaces à courbure strictement négative. On s'attend donc à ce que le flot géodésique ait un comportement fortement stochastique sur ces variétés. Il existe plusieurs approches décrivant le caractère "chaotique" du flot, d'un point de vue quantitatif.

- On peut chercher à étudier le problème de l'ergodicité relativement au volume riemannien. Lorsque la variété est compacte, la mesure de Liouville, définie sur le fibré unitaire de la variété, est une mesure finie invariante par le flot géodésique. C'est sans doute la mesure naturelle du point de vue géométrique.

- On peut vouloir au contraire étudier les trajectoires du flot qui sont les plus désordonnées possibles, au sens probabiliste du terme. Il s'agit dans ce cas de construire une mesure invariante qui maximise l'entropie. 
- Enfin, on peut se demander si l'ergodicité est une propriété typique des mesures invariantes par le flot. C'est le point de vue générique. L'ensemble des mesures invariantes est muni d'une topologie naturelle, et il s'agit de montrer que l'ensemble des mesures ergodiques de support total est un "gros" ensemble, au sens de Baire.

Il nous a semblé intéressant de confronter ici ces trois approches, dans la mesure où elles font appel à des méthodes de nature assez différente, et pourtant s'éclairent mutuellement.

$\mathrm{Du}$ reste, on ne saurait comprendre les propriétés quantitatives du flot géodésique, sans s'intéresser tout d'abord à ses propriétés qualitatives, c'està-dire à sa récurrence topologique et à sa transitivité.

Pour cette raison, les deux premières parties de cet article sont consacrées à des questions de dynamique topologique. La situation est relativement bien comprise sur les variétés compactes, mais il y a peu de résultats en présence de trajectoires errantes. On a surtout cherché à mettre en valeur les différences qui existent entre la courbure strictement négative et la courbure négative ou nulle.

Les deux dernières parties portent sur les propriétés quantitatives du flot géodésique, défini sur une variété de rang un. On présente trois points de vue: d'abord les travaux de Y. Pesin concernant l'ergodicité du flot relativement au volume; ensuite les résultat de G. Knieper relatifs à l'entropie; enfin l'approche générique que nous avons adoptée dans un travail en commun avec B. Schapira.

On a donné quelques preuves afin d'illustrer les méthodes et les idées les plus intéressantes. Certaines de ces preuves sont nouvelles, d'autres au contraire sont classiques et jouent un rôle important dans la théorie. Enfin ce texte est parsemé de questions ouvertes, certaines sans doute faciles, d'autres plus difficiles, dans l'espoir qu'elles suscitent l'intérêt du lecteur pour un sujet qui recèle bien des mystères.

\section{Quelques PROPRIÉTÉS DE LA COURBURE NÉGATIVE OU NULLE}

\subsection{NOTATIONS}

Dans la suite, $M$ est une variété riemannienne $C^{3}$ connexe complète à courbure négative ou nulle, de dimension supérieure ou égale à deux. Son fibré unitaire est noté $X$ ou $T^{1} M$ en fonction du contexte. Le revêtement universel de $M$ est noté $\widetilde{M}$. Enfin, $\widetilde{X}$ est le fibré unitaire de $\widetilde{M}$. 
La métrique riemannienne sur $M$ induit une métrique sur son fibré unitaire $X$, appelée métrique de Sasaki et définie comme suit: étant donné un vecteur $\mathcal{X} \in T X$ et une courbe $V:]-\varepsilon, \varepsilon[\rightarrow X$ tangente à ce vecteur en 0 , la norme de $\mathcal{X}$ est donnée par la formule

$$
\|\mathcal{X}\|_{X}^{2}=\left\|c^{\prime}(0)\right\|^{2}+\left\|\nabla_{c^{\prime}} V(0)\right\|^{2}
$$

où $c$ est la courbe obtenue en projetant $V$ sur $M$ et $\nabla_{c^{\prime}} V(0)$ est la dérivée covariante de $V$ le long de $c$ en 0 . Pour cette métrique, les fibres de la projection de $X$ sur $M$ sont totalement géodésiques, tandis que la courbe formée par les vecteurs unitaires tangents à une géodésique de $M$ est une géodésique de $X$.

Nous dirons que la courbure est strictement négative $(K<0)$, si toutes les courbures sectionnelles, en tout point de la variété, sont strictement négatives. On parlera de courbure négative pincée si les courbures sectionnelles sont toutes comprises entre deux nombres strictement négatifs.

Nous allons étudier la dynamique du flot géodésique $g_{t}: X \rightarrow X$. Pour alléger les notations, le relevé du flot à $\widetilde{M}$ est aussi noté $g_{t}$. Le terme "géodésique" est utilisé aussi bien pour désigner les orbites du flot géodésique sur $X$ que leurs projections sur $M$.

\subsection{VARIÉTÉS DE HADAMARD}

Rappelons quelques propriétés élémentaires des variétés de courbure négative ou nulle.

THÉORÈME 1.1 (J. Hadamard). Soit $x_{0} \in M$; l'application exponentielle exp: $T_{x_{0}} M \rightarrow M$ est un revêtement.

Une variété riemannienne connexe complète à courbure négative ou nulle est appelée variété de Hadamard si elle est simplement connexe. Le résultat précédent montre que toute variété de Hadamard de dimension $n$ est difféomorphe à $\mathbf{R}^{n}$.

Le revêtement universel $\widetilde{M}$ de $M$ est une variété de Hadamard, et il peut être identifié à $T_{x_{0}} M$ ou $T_{x_{0}} \widetilde{M}$ par le biais de l'application exponentielle. Comme les géodésiques issues de $x_{0}$ se relèvent en des droites de $T_{x_{0}} \widetilde{M}$, nous voyons que par deux points distincts de $\widetilde{M}$ passe une unique géodésique.

\subsection{Convexité}

Introduisons la notion de sous-ensemble convexe d'une variété de Hadamard. Un sous-ensemble $C \subset \widetilde{M}$ est convexe si pour tous points $x, y$ distincts de $C$, 
la géodésique qui relie $x$ à $y$ est incluse dans $C$.

On dispose d'un théorème de projection sur les convexes fermés: soit $C$ un convexe fermé dans une variété de Hadamard et $x$ un point extérieur à ce convexe, alors il existe un unique point dans $C$ qui réalise la distance de $x$ à $C$. Ce point est appelé projection de $x$ sur $C$, et la géodésique qui relie $x$ à son projeté est orthogonale au convexe. La fonction distance entre deux géodésiques est aussi convexe:

THÉORÈME 1.2. Soient $c_{1}, c_{2}: \mathbf{R} \rightarrow \widetilde{M}$ deux géodésiques dans une variété de Hadamard $\widetilde{M}$. Alors la fonction

$$
t \mapsto d\left(c_{1}(t), c_{2}(t)\right)
$$

est convexe.

Le comportement asymptotique d'une fonction convexe à l'infini est relativement simple, si bien que ce théorème permet de déduire des informations sur les géodésiques de $\widetilde{M}$. Soient $c_{1}, c_{2}: \mathbf{R} \rightarrow \widetilde{M}$ deux géodésiques géométriquement distinctes, c'est-à-dire telles que $c_{1}(\mathbf{R}) \neq c_{2}(\mathbf{R})$. Considérons la fonction $t \mapsto d\left(c_{1}(t), c_{2}(t)\right)$. Cette fonction:

- a un unique minimum: les deux géodésiques ont au plus un point en commun; si $c_{1}(\mathbf{R}) \cap c_{2}(\mathbf{R})$ est vide, il y a une géodésique de longueur minimale qui relie $c_{1}$ à $c_{2}$, elle est orthogonale à $c_{1}$ et $c_{2}$ aux points d'intersection;

- est constante (ou bornée sur $\mathbf{R}$ ): les deux géodésiques bordent une sousvariété à bord euclidienne totalement géodésique isométrique à $[0, a] \times \mathbf{R}$ (théorème de la bande plane) [BGS85, p.17]. La courbure sectionnelle s'annule donc sur les plans tangents à cette sous-variété et ce cas est exclu en courbure strictement négative;

- n'a pas de minimum:

- la borne inférieure est nulle: si cette borne inférieure est atteinte en $+\infty$, la fonction $d\left(c_{1}(t), c_{2}(t)\right)$ décroît vers 0 lorsque $t$ tend vers $+\infty$. On dit alors que $c_{1}^{\prime}(0)$ est sur la variété stable de $c_{2}^{\prime}(0)$. Dans le cas contraire, elle décroît vers 0 quand $t$ tend vers $-\infty$, et $c_{1}^{\prime}(0)$ est sur la variété instable de $c_{2}^{\prime}(0)$. Ce cas ne se produit pas si la courbure est nulle partout;

- la borne inférieure est strictement positive : la situation est plus complexe. A reparamétrage près, on peut être dans la situation précédente. Ou encore, les deux géodésiques peuvent se rapprocher d'une bande plane lorsque $t$ tend vers l'infini. Voici un petit exercice 
en dimension deux qui traite le cas où une des deux géodésiques est périodique dans un certain quotient de $\widetilde{M}$;

et ces cas sont mutuellement exclusifs.
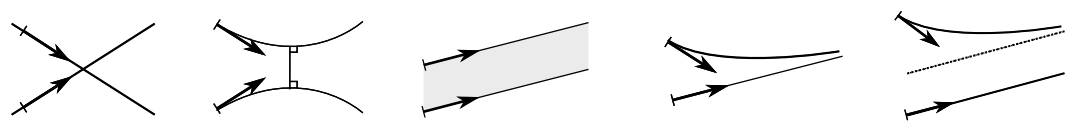

EXERCICE. On se place dans le dernier cas considéré : $t \mapsto d\left(c_{1}(t), c_{2}(t)\right)$ n'a pas de minimum sur $\mathbf{R}$ et sa borne inférieure est non nulle. Supposons $\widetilde{M}$ de dimension deux. S'il existe un réel $l \neq 0$ et une isométrie $\gamma$ de $\widetilde{M}$ telle que $\gamma\left(c_{2}(t)\right)=c_{2}(t+l)$ pour tout $t \in \mathbf{R}$, alors $c_{1}$ est sur la feuille stable d'une géodésique qui borde une bande plane avec $c_{2}$.

Dans ce cas, l'image de la bande plane dans $\widetilde{M} /\langle\gamma\rangle$ est un cylindre ou un ruban de Moebius. La situation est plus subtile en dimension supérieure.

EXEMPLE. Considérons une isométrie $\gamma$ de $\widetilde{M}=\mathbf{R}^{2} \times \mathbf{R}$ qui est le produit d'une rotation irrationnelle par une translation. La droite verticale $\mathcal{D}=\{0\} \times \mathbf{R}$ passant par l'origine de $\widetilde{M}$ se projette dans $\widetilde{M} /\langle\gamma\rangle$ sur une géodésique périodique. Considérons maintenant une autre droite verticale dans $\widetilde{M}$; elle délimite avec $\mathcal{D}$ une bande plane dans $\widetilde{M}$, mais sa projection n'est pas périodique, elle est dense dans un tore de la forme $S^{1} \times S^{1}$.

Du point de vue du flot géodésique sur le fibré unitaire de $\widetilde{M} /\langle\gamma\rangle$, il y a trois types d'orbites: les deux orbites périodiques qui passent par l'origine, les orbites issues des vecteurs verticaux qui sont récurrentes, et les autres orbites, qui partent toutes à l'infini, aussi bien pour les temps positifs que pour les temps négatifs.

Remarquons que dans le quotient $\widetilde{M} /\langle\gamma\rangle$, l'ensemble des géodésiques fermées n'est pas dense dans l'ensemble des géodésiques récurrentes.

\section{DifFÉRENCES ENTRE $K<0$ ET $K \leq 0$}

Les premières différences entre la courbure strictement négative et la courbure négative ou nulle apparaissent au niveau des variétés stables. 


\subsection{VARIÉTÉS STABLES : DÉFINITION DYNAMIQUE}

Soit $v$ un point de $X$ ou $\widetilde{X}$. La variété stable de $v$ est définie par:

$$
W^{s s}(v)=\left\{w \in X \mid d\left(g_{t}(v), g_{t}(w)\right) \rightarrow 0 \text { quand } t \rightarrow+\infty\right\} .
$$

Cette définition n'est pas spécifique au flot géodésique, elle reste valide pour n'importe quel flot défini sur un espace métrique. De manière abusive, on parlera de feuilletage stable pour désigner la partition de $X$ en feuilles stables. Illustrons par un exemple comment utiliser ce concept de variété stable pour étudier la dynamique du flot.

\section{ApPliCATION. Transitivité du flot.}

Nous allons voir que si les feuilles stables et instables couvrent suffisamment d'espace, alors le flot $g_{t}$ possède une orbite dense dans $X$. Rappelons la définition d'un point récurrent sous l'action d'un flot continu $g_{t}$ défini sur un espace métrique $X$.

DÉfINITION. Nous dirons que $v \in X$ est positivement (resp. négativement) récurrent, si on peut trouver $t_{n} \rightarrow+\infty$ (resp. $\left.-\infty\right)$ tel que $g_{t_{n}}(v) \rightarrow v$. Nous dirons que $v$ est récurrent s'il est à la fois positivement et négativement récurrent.

Proposition 2.1. Soit $g_{t}$ un flot continu défini sur un espace métrique, soit $U$ un ouvert invariant par $g_{t}$ et $v$ un point de $U$ qui est récurrent. Alors la feuille stable de $v$ est incluse dans $U: W^{s s}(v) \subset U$.

Preuve. Soit $w \in W^{s s}(v)$. Alors

$$
d\left(g_{t_{n}}(w), v\right) \rightarrow 0 \text {. }
$$

On peut trouver $n$ tel que $g_{t_{n}}(w)$ est dans $U$, et comme l'ouvert $U$ est invariant par $g_{t}$, le point $w$ est dans $U$. La proposition est démontrée.

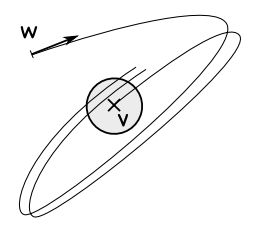

Avec un peu de régularité sur $W^{s s}$, on démontre un résultat plus précis. Pour cela, nous avons besoin de quelques rappels élémentaires sur les relations d'équivalence.

Le feuilletage stable définit une relation d'équivalence sur $X$ : deux points sont équivalents si l'un est sur la feuille stable de l'autre. Le saturé d'un ensemble par cette relation d'équivalence est l'union de toutes les feuilles stables qui rencontrent cet ensemble. Nous dirons que le feuilletage stable 
définit une relation d'équivalence ouverte si pour tout ouvert $U$ de $X$, le saturé de $U$ est ouvert. Cette propriété est vérifiée, par exemple, si $X$ est une variété et si les feuilles stables définissent un authentique feuilletage continu.

Les relations d'équivalence ouvertes possèdent la propriété suivante: le saturé de l'adhérence d'un ensemble quelconque est contenu dans l'adhérence de son saturé. Cette propriété est laissée en exercice, elle permet de démontrer la proposition suivante:

PROPOSITION 2.2. Soit $g_{t}$ un flot continu défini sur un espace métrique $X$. On suppose que la relation d'équivalence associée au feuilletage stable est ouverte, et que les points récurrents sont denses dans $X$. Soit $U$ un ouvert de $X$ invariant par $g_{t}$. Alors l'adhérence de $U$ est saturée par les feuilles stables : $\forall v \in \bar{U}, W^{s s}(v) \subset \bar{U}$.

Preuve. Notons $\mathcal{R}$ l'ensemble des points récurrents, et pour tout $A \subset X$, notons $R(A)$ le saturé de $A$ par les feuilles stables: $R(A)=\bigcup_{v \in A} W^{s s}(v)$. On a alors :

$$
R(\bar{U})=R(\overline{U \cap \mathcal{R}}) \subset \overline{R(U \cap \mathcal{R})} \subset \bar{U} .
$$

La proposition est établie.

Renversons maintenant le sens du temps. La feuille instable d'un point $v \in X$ est définie comme suit:

$$
W^{s u}(v)=\left\{w \in X \mid d\left(g_{t}(v), g_{t}(w)\right) \rightarrow 0 \text { quand } t \rightarrow-\infty\right\}
$$

Supposons que la relation associée au feuilletage instable est ouverte, et que les points négativement récurrents sont denses dans $X$. Alors l'adhérence de tout ouvert invariant par $g_{t}$ contient toutes les feuilles instables qui intersectent cet ouvert.

Supposons de plus qu'en tout point $v \in X$, on peut trouver un voisinage dont tous les points peuvent être reliés à $v$ par un chemin composé d'un nombre fini de morceaux, chacun inclus dans une feuille stable, une feuille instable ou une orbite du flot.

Alors l'adhérence de tout ouvert invariant non vide

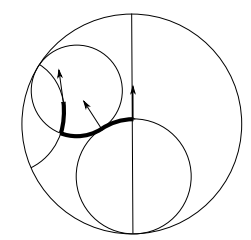
$U$ est un ensemble ouvert. Si $X$ est connexe, on en déduit que $U$ est dense dans $X$ : c'est la transitivité du flot sur $X$. 
RAPPEL. Soit $X$ un espace métrique séparable possédant la propriété de Baire, $g_{t}: X \rightarrow X$ un flot continu. Supposons que tout ouvert non vide invariant par le flot est dense dans $X$. Alors il existe un point dont l'orbite sous l'action $d u$ flot est dense dans $X$.

Preuve. Les points dont l'orbite est dense sont précisément les éléments de l'ensemble:

$$
\bigcap_{U}\left\{g_{t}(v) \mid v \in U, t \in \mathbf{R}\right\}
$$

où l'intersection a lieu sur toutes les boules ouvertes $U$ non vides, de rayon rationnel, avec un centre appartenant à une partie dénombrable dense fixée à l'avance. Comme chacun des $g_{\mathbf{R}}(U)$ est dense, on peut appliquer le théorème de Baire. Ceci termine la preuve.

Revenons au cas du flot géodésique, défini sur le fibré unitaire $X=T^{1} M$ de $M$. Les hypothèses précédentes s'appliquent dès que la variété $M$ est à courbure strictement négative pincée. On retrouve un théorème bien connu.

THÉORÈME 2.3. On suppose que $M$ est à courbure strictement négative pincée, et que les vecteurs récurrents sous l'action du flot géodésique sont denses dans $T^{1} M$. Alors le flot géodésique est transitif.

La densité des points récurrents est par exemple vérifiée en volume fini, c'est une conséquence du théorème de récurrence de Poincaré. En courbure constante, volume fini, la transitivité du flot géodésique remonte aux travaux de E. Artin, J. Nielsen, P. Koebe, F. Löbell et M. Morse dans les années trente.

\subsection{HOROSPHÈRES: DÉFINITION GÉOMÉTRIQUE}

Commençons par définir le concept d'horosphère. Considérons un vecteur $v \in \widetilde{X}$, et notons $\pi: \widetilde{X} \rightarrow \widetilde{M}$ la projection du fibré unitaire sur la variété. L'horoboule basée en $v$ est le sous-ensemble de $\widetilde{M}$ union de toutes les boules ouvertes de rayon $t$ centrées en $\pi\left(g_{t}(v)\right)$ :

$$
H B(v)=\bigcup_{t \geq 0} B\left(\pi\left(g_{t}(v)\right), t\right)
$$

C'est un ensemble convexe dont le bord contient $\pi(v)$.

De manière générale, le bord d'un convexe possède une certaine forme de régularité. Par exemple, en dimension deux, il est forcément continu, avec une dérivée à gauche et à droite en chacun de ses points, et ces deux dérivées coïncident hormis en un nombre dénombrable de points. 
Une étude plus détaillée montre que la convergence des boules est en fait $C^{1}$ [BGS85, 3.4], et même $C^{2}$ [H-IH77] (mais pas mieux [BBB87]), si bien que le bord de l'horoboule est une sous-variété lisse en tout point: c'est l'horosphère associée au vecteur $v$. Cette horosphère se relève au fibré unitaire $\widetilde{X}$ de $\widetilde{M}$ : l'horosphère stable basée en $v$ est composée de tous les vecteurs de $\widetilde{X}$ basés sur le bord de l'horoboule, qui sont orthogonaux à ce bord, et orientés vers l'intérieur de l'horoboule. On la note $\operatorname{Hor}^{+}(v)$. On peut projeter ces horosphères sur $X$ pour obtenir un feuilletage, dont la régularité n'est à priori pas meilleure que $C^{0}$.

L'horosphère instable basée en un point $v \in \widetilde{X}$ est définie en considérant l'horoboule associée à $-v$ et en prenant les vecteurs de $\widetilde{X}$ qui sont orthogonaux au bord de cette horoboule, dirigés vers l'extérieur de l'horoboule.
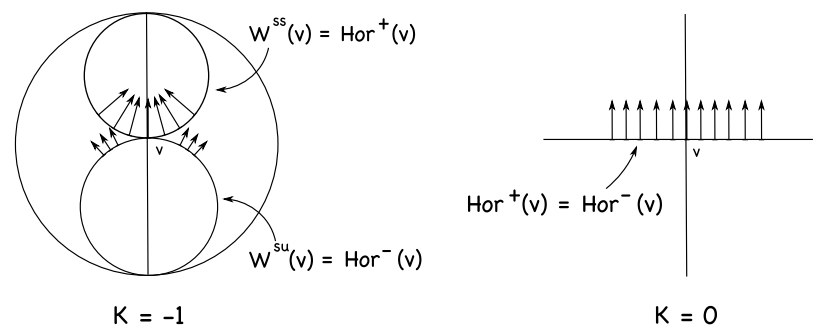

Décrivons brièvement ces horosphères dans le cas d'une variété de courbure constante. Si la courbure est strictement négative, on peut se placer dans le modèle de la boule de Poincaré; les horosphères sont des sphères qui sont tangentes au bord de la boule. Dans l'espace euclidien, les horosphères sont des hyperplans. Dans ces deux cas, ce sont des objets réguliers qui définissent un feuilletage $C^{\infty}$. C'est une situation exceptionnelle:

ThÉorème 2.4 (G. Besson, G. Courtois, S. Gallot [BCG95]). Soit M une variété compacte $C^{\infty}$ à courbure strictement négative, telle que les feuilletages horosphériques stables et instables sont $C^{\infty}$. Alors $M$ est localement symétrique.

On verra pourquoi la question de la régularité du feuilletage horosphérique est importante, lorsqu'on abordera le problème de l'ergodicité du flot géodésique.

Remarquons pour terminer qu'en courbure strictement négative, les horosphères stables et instables sont transverses. En courbure nulle, par contre, l'horosphère stable et l'horosphère instable basée en un point quelconque de $\widetilde{M}$ coïncident. 
APPLICATION. Densité des horosphères.

Nous allons illustrer, à partir de la définition géométrique des horosphères, comment on peut étudier le problème de leur densité. Introduisons deux nouveaux concepts.

Une géodésique $c: \mathbf{R} \rightarrow M$ est quasi-minimisante si on peut trouver une constante $C>0$ telle que pour tout $t \geq 0, d(c(0), c(t)) \geq t-C$.

Soit $\Gamma$ le groupe fondamental de $M$, identifié à un sous-groupe des isométries de $\widetilde{M}$ : $\widetilde{M} / \Gamma \simeq M$. Une géodésique $\tilde{c}: \mathbf{R} \rightarrow \widetilde{M}$ n'est pas de type horosphérique si on peut trouver un $t_{0} \geq 0$ tel que $\Gamma . \tilde{c}(0) \cap \operatorname{Hor}^{+}\left(\tilde{c}^{\prime}\left(t_{0}\right)\right)=\varnothing$. Un vecteur $v \in X$ est dit de type horosphérique s'il admet un relevé à $\widetilde{X}$ qui engendre une géodésique de type horosphérique, auquel cas

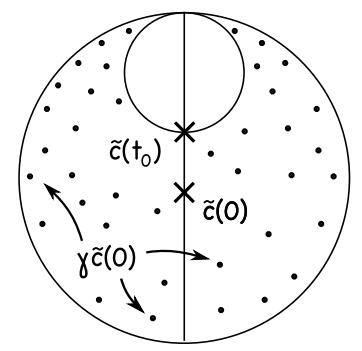
tous ses relevés engendrent de telles géodésiques.

Le théorème suivant est valide en courbure négative ou nulle.

THÉORÈME 2.5 (P. Eberlein [Eb73]). Une géodésique de $M$ est quasiminimisante si et seulement si son relevé à $\widetilde{M}$ n'est pas horosphérique.

Preuve.

Non horosphérique $\Leftrightarrow \quad \forall t \geq 0, \quad \Gamma . \tilde{c}(0) \cap B\left(\tilde{c}\left(t_{0}+t\right), t\right)=\varnothing$,

$$
\begin{aligned}
& \Leftrightarrow \quad \forall t \geq 0, \quad \forall \gamma \in \Gamma, d\left(\gamma \tilde{c}(0), \tilde{c}\left(t_{0}+t\right)\right) \geq t \quad \text { sur } \tilde{M}, \\
& \Leftrightarrow \quad \forall t \geq 0, \quad d(c(0), c(t)) \geq t-t_{0} \quad \text { sur } M .
\end{aligned}
$$

Ceci termine la preuve.

En courbure strictement négative pincée, sous la condition que les orbites récurrentes du flot géodésique sont denses dans $X$, on démontre que tout vecteur de type horosphérique est sur une horosphère qui est dense dans $X$. Ce résultat est dû à $\mathrm{P}$. Eberlein [Eb73], on trouvera des généralisations dans l'article de F. Dal'bo [D00] et dans [C05]. En résumé,

THÉORÈME 2.6. Supposons que $M$ est à courbure strictement négative pincée et que les orbites récurrentes du flot géodésique sont denses dans $X=T^{1} M$. Considérons un vecteur $v \in X$. Alors l'horosphère stable basée en $v$ est dense dans $X$ si et seulement si la géodésique sur $M$ engendrée par ce vecteur n'est pas quasi-minimisante. 
Ce résultat implique par exemple que le feuilletage horosphérique est minimal (i.e. toutes les horosphères stables sont denses dans $M$ ) si et seulement si $M$ est compact.

EXERCICE. Construire une surface à courbure négative pincée, et un vecteur sur cette surface, tels que l'horosphère stable associée à ce vecteur est dense dans la surface, mais le vecteur part à l'infini sous l'action du flot géodésique.

\subsection{VARIÉTÉS STABLES ET HOROSPHÈRES}

De manière générale, l'horosphère stable basée en un vecteur $v \in \widetilde{X}$ contient toujours la variété stable de $v: W^{s s}(v) \subset \operatorname{Hor}^{+}(v)$. De plus, variétés stables et horosphères stables coïncident en courbure strictement négative: $W^{s s}(v)=\operatorname{Hor}^{+}(v)$. Par contre elles sont en général distinctes si la courbure s'annule. Donnons quelques exemples.

Pour les variétés à courbure nulle comme le tore plat $\mathbf{R}^{2} / \mathbf{Z}^{2}$, les feuilles stables sont réduites à des points tandis que les horosphères sont localement des hyperplans. Horosphères stables et instables coïncident, si bien qu'on ne peut pas joindre deux points quelconques de la variété par un chemin composé de morceaux de feuilles stables, de feuilles instables et de géodésiques. Le flot géodésique n'est du reste pas transitif.

Plaçons nous maintenant sur une surface à courbure négative ou nulle qui contient un cylindre euclidien $[0,1] \times S^{1}$. Les feuilles stables associées aux vecteurs qui engendrent une géodésique périodique contenue dans l'intérieur du cylindre sont triviales (i.e. $W^{s s}(v)=\{v\}$ ). De fait, la géodésique engendrée par un vecteur qui se trouve sur la feuille stable d'un tel point, doit rester dans le cylindre à partir d'un temps suffisamment grand. Mais seules les géodésiques périodiques dans le cylindre possèdent cette propriété, toutes les autres traversent le cylindre de part en part. Pour ce qui est des horosphères stables associées aux vecteurs dont la trajectoire est périodique, contenues dans le cylindre, ce sont des sous-variétés immergées de dimension un. Les horosphères instables coïncident avec les horosphères stables dans un voisinage du vecteur, et ne se différencient de celles-ci qu'à la sortie du cylindre.

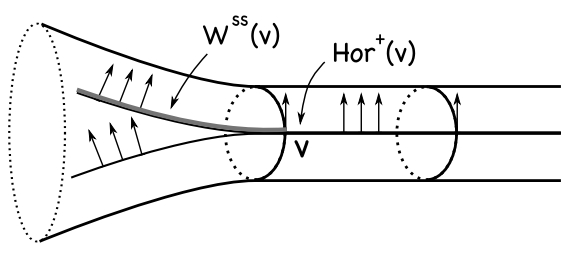


Donnons un exemple où la feuille stable est non triviale, sans pour autant coïncider avec l'horosphère stable. Considérons une géodésique périodique définie sur une surface orientable, qui borde d'un côté un cylindre euclidien, et telle que la courbure soit strictement négative de l'autre côté, au voisinage de la géodésique. Soit $v$ un vecteur sur cette géodésique. Au voisinage de $v$, la feuille stable de $v$ coïncide avec la partie de l'horosphère qui se trouve du côté où la courbure est strictement négative. La feuille est donc égale à une demi-horosphère.

\subsection{LIEN AVEC LES SYSTÈMES HYPERBOLIQUES}

Un flot géodésique défini sur le fibré unitaire d'une variété compacte de courbure strictement négative est un flot d'Anosov: le fibré tangent $T X$ est la somme directe de trois fibrés $E^{0}, E^{s}, E^{u}$ invariants par la différentielle du flot, qui satisfont pour des constantes $C, \lambda>0$,

- $E^{0}$ est tangent à la direction du flot,

- $\forall v \in E^{s}, \forall t>0,\left\|d g_{t} v\right\| \leq C e^{-\lambda t}\|v\|$,

- $\forall v \in E^{u}, \forall t>0,\left\|d g_{-t} v\right\| \leq C e^{-\lambda t}\|v\|$.

Dans le cas du flot géodésique, les espaces $E^{s}$ et $E^{u}$ sont les espaces tangents aux horosphères stables et instables. On parle d'hyperbolicité uniforme pour qualifier ce genre de propriétés. La plupart des propriétés dynamiques du flot géodésique en courbure strictement négative découle de cette hyperbolicité uniforme.

Il existe des variétés compactes, à courbure négative ou nulle, sur lesquelles le flot géodésique est encore Anosov. Dans ce cas, le flot possède des propriétés similaires à celles observées en courbure strictement négative. La distinction, du point de vue dynamique, n'est pas tant entre courbure strictement négative et courbure négative ou nulle, mais plutôt entre flot géodésique Anosov et non Anosov.

À quelles conditions sur la courbure, le flot géodésique est-il Anosov?

Donnons tout d'abord une obstruction élémentaire à la propriété d'Anosov. Les flots d'Anosov possèdent en chaque point des variétés stables et instables qui forment des sous-variétés de dimension non nulle transverses à la direction du flot. Par conséquent, si un vecteur possède une variété stable triviale ( $W^{s s}(v)=\{v\}$ ), le flot géodésique ne peut pas être Anosov.

Considérons une surface compacte à courbure négative ou nulle qui contient un compact isométrique à un cylindre euclidien $[0,1] \times S^{1}$. Les vecteurs dans le cylindre qui sont verticaux, c'est-à-dire, qui sont tangents à un méridien $\{x\} \times S^{1}$, ont une variété stable triviale. De fait, une trajectoire asymptotique 
à un tel vecteur doit rester dans le cylindre pour tous les temps suffisamment grands. Mais dans le cylindre, les trajectoires sont des droites euclidiennes, elles traversent donc le cylindre de part en part, hormis pour celles qui sont verticales. Le flot ne peut donc pas être Anosov.

Le critère suivant, dû à $\mathrm{P}$. Eberlein, donne des exemples de surfaces où la courbure s'annule, mais pour lesquelles le flot géodésique est Anosov.

THÉORÈME 2.7 (P. Eberlein). Soit $M$ une surface compacte à courbure négative ou nulle. Le flot géodésique défini sur $T^{1} M$ est Anosov si et seulement si on peut trouver sur chaque géodésique un point de courbure non nulle.

On peut aussi chercher à établir de l'hyperbolicité uniforme dans le cas non compact. Les zones qui posent problème sont alors celles constituées par des géodésiques récurrentes le long desquelles la courbure est toujours nulle.

EXERCICE. Calculer la courbure de la surface:

$$
\left\{(x, y, z) \in \mathbf{R}^{3} /(2 \pi \mathbf{Z})^{3} \mid \cos (x)+\cos (y)+\cos (z)=0\right\} .
$$

En déduire que le flot géodésique, défini sur le fibré unitaire de cette surface, est Anosov. Donnez un exemple de surface admettant des points où la courbure est strictement positive, mais pour laquelle le flot géodésique est Anosov.

\subsection{ENSEMBLE NON ERRANT}

On s'intéresse maintenant au cas où les points récurrents ne sont pas denses dans la variété. Rappelons la définition de l'ensemble non errant.

DÉFINITION. Soit $g_{t}: X \rightarrow X$ un flot défini sur un espace métrique. Son ensemble non errant est défini comme suit:

$$
\Omega=\left\{x \in X \mid \forall \epsilon>0, \exists t_{n} \rightarrow+\infty \text { tel que } g_{t_{n}}(B(x, \epsilon)) \cap B(x, \epsilon) \neq \varnothing\right\} .
$$

Voici quelques propriétés générales de l'ensemble non errant $\Omega$.

- L'ensemble non errant est fermé, invariant par le flot.

- Les points récurrents appartiennent à $\Omega$.

- Les mesures de probabilité invariantes ont leurs supports inclus dans $\Omega$.

Considérons le cas du flot géodésique défini sur le fibré unitaire d'une variété à courbure négative ou nulle. Lorsque la courbure est strictement 
négative, on a le résultat suivant:

THÉORÈME 2.8. Supposons $M$ à courbure strictement négative. Alors les géodésiques fermées sont denses dans l'ensemble non errant. Supposons de plus que $\Omega$ contient plus de deux géodésiques périodiques. Alors le flot géodésique $g_{t}$ est transitif en restriction à cet ensemble: il existe un vecteur $v \in \Omega$ tel que $\left\{g_{t}(v) \mid t \in \mathbf{R}\right\}$ est dense dans $\Omega$.

Le cas d'un cylindre à courbure -1 donne un exemple pour lequel $\Omega$ contient exactement deux orbites périodiques; ces orbites sont engendrées par un vecteur et son opposé. Le flot n'est bien sûr pas transitif sur $\Omega$ dans ce cas.

Le théorème précédent est pris en défaut en courbure négative ou nulle. On a donné un exemple d'un cylindre de dimension trois possédant une seule géodésique périodique (deux en comptant le sens de parcours), et une infinité de géodésiques récurrentes.

Décrivons un exemple non trivial de surface à courbure négative ou nulle, sur laquelle le flot géodésique n'est pas transitif en restriction à l'adhérence des orbites périodiques. Cette surface est difféomorphe au tore privé d'un point. Elle admet une géodésique périodique $c$ qui sépare la surface en deux parties. La partie qui se trouve à gauche sur le dessin est compacte à courbure strictement négative. La partie droite est isométrique à un demicylindre euclidien $\left[0,+\infty\left[\times S^{1}\right.\right.$.

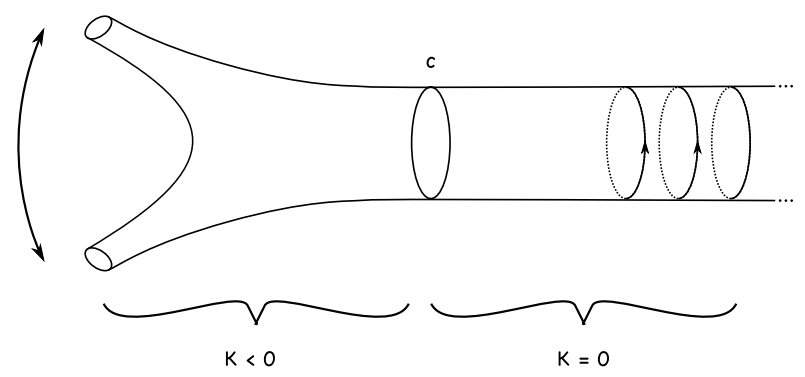

Il existe trois types différents d'orbites sur le fibré unitaire de cette surface.

- les orbites périodiques contenues dans le cylindre euclidien,

- les orbites qui restent dans la partie à courbure strictement négative,

- les orbites qui croisent la géodésique $c$. Elles admettent un voisinage constitué de vecteurs dont la trajectoire tend vers $+\infty$, pour les temps positifs ou pour les temps négatifs. Ces orbites sont errantes. 
L'ensemble non errant $\Omega$ contient toutes les orbites périodiques. Son intersection avec le demi-cylindre est composé exactement des orbites périodiques contenues dans le cylindre. Une trajectoire qui approche une de ces orbites périodiques sans être elle même périodique doit être errante, elle ne peut donc pas être récurrente ni dense dans $\Omega$. Le flot n'est pas transitif en restriction à $\Omega$, même si les orbites périodiques sont denses dans l'ensemble non errant.

\section{QUESTIONS.}

- Quelles sont les surfaces à $K \leq 0$ pour lesquelles le flot géodésique est transitif sur $\Omega$ ?

- Quelles sont les surfaces à $K \leq 0$ pour lesquelles les géodésiques périodiques sont denses dans $\Omega$ ?

On connaît la réponse à ces questions lorsque $\Omega=X$. Le théorème suivant traite le cas de la dimension deux.

THÉORÈME 2.9 (P. Eberlein [Eb96]). Soit $M$ une surface à courbure négative ou nulle, avec au moins un point de courbure strictement négative, telle que $\Omega=T^{1} M$. Alors les orbites périodiques sont denses dans $T^{1} M$ et le flot géodésique est transitif sur $T^{1} M$.

Le cas de la dimension supérieure sera mentionné plus loin.

En courbure négative ou nulle, il existe peu de résultats sur la dynamique topologique du flot géodésique, dans le cas où $\Omega \neq X$. On peut quand même énoncer un théorème en relation avec la transitivité. Pour cela nous avons besoin du concept d'orbite périodique hyperbolique pour un flot $C^{1}$.

DÉFINITION. Soit $X$ une variété différentielle, $g_{t}: X \rightarrow X$ un flot $C^{1}$ et $v \in X$ un point périodique de période $l \neq 0$. La différentielle $d_{v} g_{l}: T_{v} X \rightarrow T_{v} X$ est une application linéaire qui admet une valeur propre égale à un; cette valeur propre est associée à la direction du flot. Nous dirons que l'orbite de $v$ est hyperbolique si les seuls vecteurs propres de cette application qui sont associés à des valeurs propres de module un sont proportionnels à la direction du flot.

En courbure strictement négative, toutes les orbites périodiques du flot géodésique sont hyperboliques. Sur une surface à courbure négative ou nulle, une géodésique périodique est hyperbolique si et seulement s'il existe un point de courbure strictement négative sur la géodésique. 
On peut à présent énoncer un résultat valide même si $\Omega$ est différent de $X$.

THÉORÈME 2.10 (Y. Coudène, B. Schapira [CS10]). Soit M une variété à courbure négative ou nulle. On suppose que l'ensemble non errant contient plus de deux géodésiques périodiques hyperboliques. Alors le flot géodésique est transitif en restriction à l'adhérence des orbites périodiques hyperboliques.

QUESTION. Pour quelles variétés à courbure négative ou nulle les géodésiques périodiques hyperboliques sont-elles denses dans $\Omega$ ?

Revenons au cas de la surface avec un bout cylindrique euclidien discuté plus haut. Les géodésiques périodiques hyperboliques sont toutes contenues dans la partie à courbure négative ou nulle, et le flot est transitif en restriction à l'adhérence de ces géodésiques. La géodésique qui borde le cylindre euclidien peut-elle être approchée par des géodésiques périodiques hyperboliques?

PROPOSITION 2.11. La géodésique qui borde la partie de la surface à courbure strictement négative est dans l'adhérence des géodésiques périodiques hyperboliques.

Preuve. Identifions le groupe fondamental de $M$ à un sous-groupe $\Gamma \mathrm{du}$ groupe d'isométries de $\widetilde{M}$ et considérons son action sur le bord du revêtement universel.

Plaçons nous sur l'espace des demi-géodésiques $r: \mathbf{R}_{+} \rightarrow \widetilde{M}$ afin de définir le bord de $\widetilde{M}$. On identifie deux demi-géodésiques $r_{1}, r_{2}$ si elles restent à distance bornée l'une de l'autre: $\exists C>0$ tel que $d\left(r_{1}(t), r_{2}(t)\right) \leq C$ pour tout $t \geq 0$. L'espace quotient obtenu est le bord idéal de $\widetilde{M}$. Fixons un point $x_{0} \in \widetilde{M}$. Le bord idéal $\partial \widetilde{M}$ est homéomorphe au cercle unité par le biais de l'application de $T_{x_{0}}^{1} \widetilde{M}$ dans $\partial \widetilde{M}$ qui associe au vecteur $v \in T_{x_{0}}^{1} \widetilde{M}$ le point du bord correspondant à la demi-géodésique issue de $v$.

Considérons l'orbite de $x_{0}$ dans $\widetilde{M} \cup \partial \widetilde{M}$ et définissons l'ensemble limite de $\Gamma$ par la formule: $\Lambda \Gamma=\overline{\Gamma x_{0}} \cap \partial \widetilde{M}$. G. Link, M. Peigné et J.-C. Picaud [LPP06] montrent que les extrémités des relevés des géodésiques périodiques hyperboliques de $M$ sont denses dans $\Lambda \Gamma \times \Lambda \Gamma$. Attention, on a vu que les géodésiques périodiques hyperboliques ne sont pas denses dans $\Omega$.

Fixons un relevé $\tilde{c}$ de la géodésique périodique $c$ qui borde la partie à courbure strictement négative. On note $c^{-}$et $c^{+}$ses deux extrémités dans $\Lambda \Gamma$. Soient $\tilde{c}_{n}$ une suite de géodésiques de $\widetilde{M}$ qui se projettent sur des géodésiques périodiques hyperboliques de $M$, et dont les extrémités $c_{n}^{-}$et $c_{n}^{+}$ 
convergent vers $c^{-}$et $c^{+}$. Quitte à passer à une sous-suite, on peut supposer que la convergence a lieu de façon monotone au voisinage de $c^{-}$et de $c^{+}$. Paramétrons $\tilde{c}_{n}$ de façon à ce que la distance de $\tilde{c}_{n}$ à $\tilde{c}(0)$ soit réalisée en $\tilde{c}_{n}(0)$.

Les géodésiques $\tilde{c}_{n}$ et $\tilde{c}$ se trouvent de part et d'autre de la géodésique $\tilde{c}_{n+1}$, si bien que la suite $d\left(\tilde{c}_{n}(0), \tilde{c}(0)\right)$ est décroissante. Soit $v$ une valeur d'adhérence de la suite $c_{n}^{\prime}(0)$. La géodésique engendrée par ce vecteur a pour extrémités $c^{-}$et $c^{+}$, elle délimite donc une bande plane avec $c$. Comme elle est dans l'adhérence des (relevés des) géodésiques périodiques hyperboliques, elle doit coïncider avec $c$ et la proposition est démontrée.

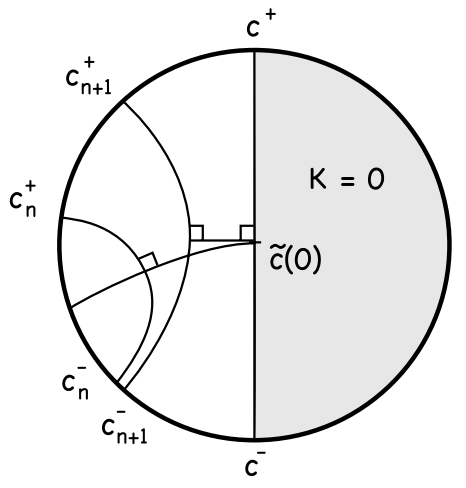

QUESTION. La feuille stable du vecteur $c^{\prime}(0)$, qui engendre la géodésique périodique sur le bord du cylindre euclidien, est-elle dense dans $\Omega$ ?

On pourra consulter [S10] pour des résultats concernant la densité des demi-horocycles en courbure strictement négative.

En résumé, on peut espérer retrouver les propriétés de la courbure strictement négative si on se restreint à un sous-ensemble de $T^{1} M$ sur lequel on a suffisamment d'hyperbolicité.

\section{VARIÉTÉS DE RANG 1}

Ceci nous amène à chercher un ensemble sur lequel la dynamique est de nature hyperbolique.

\subsection{DÉFINITION DU RANG D'UNE VARIÉTÉ}

Commençons par généraliser le concept de géodésique hyperbolique au cas non périodique. Considérons une géodésique $c: \mathbf{R} \rightarrow M$ définie sur une variété à courbure négative ou nulle. On dit qu'un champ de vecteurs $J(t): \mathbf{R} \rightarrow T M$ est défini le long de la géodésique $c$ si pour tout $t \in \mathbf{R}, J(t)$ appartient à $T_{c(t)} M$. Un tel champ est parallèle si sa dérivée covariante le long de la géodésique est nulle: $J^{\prime}(t)=\nabla_{c^{\prime}} J(t)=0$, pour tout $t \in \mathbf{R}$. 
DÉFINITION. Le rang de la géodésique $c$ est égal à 1 , plus la dimension de l'espace vectoriel des champs de vecteurs définis le long de $c$, parallèles, orthogonaux à la direction de la géodésique, et tels que la courbure s'annule dans le plan déterminé par le champ de vecteurs et la direction de la géodésique :

$$
\operatorname{rang}(c)=1+\operatorname{dim}\left\{J \mid\left\langle J(t), c^{\prime}(t)\right\rangle=0, J^{\prime}(t)=0, K\left(J(t), c^{\prime}(t)\right)=0\right\} .
$$

Une variété est dite de rang un si elle est connexe complète à courbure négative ou nulle, et si elle admet une géodésique de rang un. Si une telle variété n'admet pas de géodésique de rang un, on dit qu'elle est de rang supérieur.

Cette définition peut se formuler à l'aide de la notion de champ de Jacobi. Un champ de vecteurs $J(t)$ défini le long d'une géodésique $c_{0}$ est un champ de Jacobi s'il satisfait l'équation:

$$
J^{\prime \prime}(t)+K\left(J(t), c_{0}^{\prime}(t)\right) c_{0}^{\prime}(t)=0,
$$

l'expression $K(.,$.$) désignant ici le tenseur de courbure. D'un point de$ vue géométrique, ces champs s'obtiennent en faisant varier les géodésiques: l'équation précédente est satisfaite si et seulement si on peut trouver une famille $C^{1}$ de géodésiques $c_{s}(t)$ telle que $\frac{d c_{s}}{d s}(t)_{\mid s=0}=J(t)$.

Le rang d'une géodésique est alors égal à la dimension de l'espace vectoriel des champs de Jacobi parallèles le long de la géodésique.

\subsection{VARIÉTÉS DE RANG UN}

Sur une surface, une géodésique est de rang un si et seulement si on peut trouver un point de courbure strictement négative sur la géodésique. Dans le cas contraire, elle est de rang deux.

Une surface à courbure négative ou nulle est de rang un si et seulement s'il existe un point où la courbure est non nulle. Dans le cas compact orientable, cela est équivalent à dire que le genre est supérieur strict à un, ou encore que la surface n'est pas homéomorphe à la sphère ou au tore; c'est une application de la formule de Gauss-Bonnet.

En dimension plus grande, une géodésique qui passe par un point où toutes les courbures sectionnelles sont strictement négatives, est une géodésique de rang un. Mais il peut arriver qu'une géodésique de rang un ne passe par aucun point où toutes les courbures sectionnelles sont strictement négatives.

Rappelons qu'une sous-variété est dite totalement géodésique si toute géodésique qui part d'un point de la sous-variété, en lui étant tangente, reste 
dans la sous-variété pendant un intervalle de temps strictement positif. On peut maintenant donner la définition d'un plat: il s'agit d'une sous-variété (éventuellement à bord) totalement géodésique dont la courbure est nulle pour la métrique induite. L'exemple le plus simple de plat est donné par une portion de cylindre euclidien plongé dans une surface. Le rang d'une géodésique incluse dans un plat est au moins égal à la dimension du plat.

EXERCICE. Construire une variété compacte de rang 1 qui possède un sous-groupe de son groupe fondamental isomorphe à $\mathbf{Z}^{2}$. Une telle variété ne porte pas de métrique riemannienne à courbure strictement négative.

Le rang est bien lié à la nature hyperbolique de la dynamique du flot géodésique. Nous pouvons à présent donner les énoncés généraux de P. Eberlein.

THÉORÈME 3.1 (P. Eberlein). Sur une variété compacte à courbure négative ou nulle, le flot géodésique est Anosov si et seulement si toutes les géodésiques sont de rang un.

Soit $M$ une variété de rang un. On suppose que tous les vecteurs de $T^{1} M$ sont non errants sous l'action du flot géodésique: $\Omega=T^{1} M$. Alors les géodésiques périodiques hyperboliques sont denses dans $T^{1} M$, et le flot géodésique est transitif.

Remarquons enfin qu'en dimension deux, les géodésiques de rang un sont celles sur lesquelles il existe un point où la courbure est strictement négative. Comme la courbure est une fonction continue, ces géodésiques forment un sous-ensemble ouvert de $T^{1} M$. Ce résultat est vrai en toute dimension:

Proposition 3.2 (P. Eberlein). Soit $M$ une variété de rang un. Alors les vecteurs de rang un forment un ouvert invariant dans $T^{1} M$. Si $\Omega=M$, cet ouvert est dense.

\subsection{VARIÉTÉS DE RANG SUPÉRIEUR}

On va se restreindre dans la suite aux variétés de rang un. Ce sont celles sur lesquelles le comportement du flot géodésique est le plus proche de celui observé en courbure strictement négative. Avant cela, donnons tout de même quelques informations sur le rang supérieur. 
En rang supérieur, le flot géodésique n'est pas ergodique relativement au volume, ni transitif [BBE]. On peut avoir en tête l'exemple du tore plat $\mathbf{R}^{2} / \mathbf{Z}^{2}$; sur le fibré unitaire du tore, l'angle que forme un vecteur avec la verticale est préservé au cours du mouvement.

On peut, d'un certain point de vue, classer les espaces de rang supérieur.

THÉORÈME 3.3 (W. Ballmann [B85]). Soit $K_{0}>0$, soit $M$ une variété riemannienne connexe, complète, de volume fini, dont les courbures sectionnelles sont toutes dans l'intervalle $\left[-K_{0}, 0\right]$. On a l'alternative suivante:

- $M$ est de rang un,

- $\widetilde{M}$ est réductible (i.e. c'est un produit riemannien $M_{1} \times M_{2}$ ),

- $M$ est un espace localement symétrique (irréductible, de rang $\geq 2$ ).

Le cas réductible comprend par exemple le tore plat. Quant aux espaces localement symétriques, ce sont des quotients de groupes de Lie (connexes, irréductibles, de type non compact, de rang supérieur). A dimension donnée, il n'existe qu'une liste finie de tels groupes. Voici les plus classiques: $S L_{n+1}(\mathbf{R})$ $(n>1), \widetilde{S O}_{p, q}(\mathbf{R})(\min \{p, q\}>1), S_{2 n}(\mathbf{R})(n>1)$.

L'espace symétrique irréductible (connexe, simplement connexe, complet, à courbure négative ou nulle, de dimension $>1$ ) de plus petite dimension est $\mathrm{SL}_{3}(\mathbf{R}) / \mathrm{SO}_{3}(\mathbf{R})$. Les espaces symétriques sont homogènes et tout point dans un tel espace est inclus dans un plat dont la dimension est égale au rang de l'espace. On renvoie à la monographie [Eb96] pour plus de détails. Remarquons pour terminer que le théorème de $\mathrm{W}$. Ballmann admet des généralisations au cas $\Omega=T^{1} M[\mathrm{~B} 95]$.

\section{ERGODICITÉ DU FLOT GÉODÉSIQUE SUR LES VARIÉTÉS DE RANG 1}

Considérons un flot $g_{t}: X \rightarrow X$ mesurable préservant une mesure de probabilité $\mu$. D'après le théorème ergodique de Birkhoff, pour toute fonction $f: X \rightarrow \mathbf{R}$ de carré intégrable, on a la convergence:

$$
\text { pp. } x \in X, \quad \frac{1}{T} \int_{0}^{T} f\left(g_{t}(x)\right) d t \underset{T \rightarrow \infty}{\longrightarrow} P f(x)
$$

où $P f$ est la projection $L^{2}$ de $f$ sur le sous-espace des fonctions invariantes par $g_{t}$. Le flot est ergodique relativement à la mesure $\mu$ si la limite $P f$ est constante, auquel cas elle vaut $\int f d \mu$ : les moyennes temporelles de $f$ le long de presque toute trajectoire du flot, coüncident avec ses moyennes spatiales. 


\subsection{L'ARgumENT DE HoPF}

La démonstration de l'ergodicité du flot géodésique sur une variété de courbure négative constante et de volume fini est due à E. Hopf [Ho36]. L'argument mis au point par E. Hopf a ensuite été généralisé dans de nombreuses directions, jusqu'à devenir un principe de théorie ergodique qui ne fait plus référence au flot géodésique.

On va présenter cet argument en détail. Rappelons comment on a défini les feuilletages stables et instables d'un flot sur un espace métrique:

$$
\begin{aligned}
& W^{s s}(v)=\left\{w \in X \mid d\left(g_{t}(v), g_{t}(w)\right) \rightarrow 0 \text { quand } t \rightarrow+\infty\right\}, \\
& W^{s u}(v)=\left\{w \in X \mid d\left(g_{t}(v), g_{t}(w)\right) \rightarrow 0 \text { quand } t \rightarrow-\infty\right\} .
\end{aligned}
$$

On dira qu'une fonction $f$ est invariante par le feuilletage stable, ou encore $W^{s s}$-invariante, si elle est constante le long des feuilles stables du flot: $\forall v, w \in X, w \in W^{s s}(v)$ implique $f(w)=f(v)$. On dira qu'une fonction est $W^{s s}$-invariante mod 0 si elle coïncide avec une fonction $W^{s s}$-invariante hors d'un ensemble négligeable.

L'ARgument DE Hopf. Soit $X$ un espace métrique, $g_{t}: X \rightarrow X$ un flot borélien qui préserve une mesure de probabilité borélienne. Alors toute fonction $L^{2}$ invariante par le flot est $W^{s s}$-invariante mod 0 .

Preuve. Supposons pour commencer que $f: X \rightarrow \mathbf{R}$ est Lipschitzienne bornée. Soit $X_{f} \subset X$ le sous-ensemble des points de $X$ de mesure totale sur lequel on a:

$$
\forall v \in X_{f}, \quad \frac{1}{t} \int_{0}^{t} f\left(g_{s}(v)\right) d s \underset{t \rightarrow+\infty}{\longrightarrow} P f(v)
$$

où $P f$ est la projection orthogonale de $f$ sur $\left\{h \in L^{2} \mid \forall t \in \mathbf{R}, h \circ g_{t}=h\right\}$.

Soit $K$ la constante de Lipschitz de $f$. Pour tout $v, w \in X_{f}$ tels que $w \in W^{s s}(v)$,

$$
\left|\frac{1}{t} \int_{0}^{t} f\left(g_{s}(v)\right) d s-\frac{1}{t} \int_{0}^{t} f\left(g_{s}(w)\right) d s\right| \leq \frac{K}{t} \int_{0}^{t} d\left(g_{s}(v), g_{s}(w)\right) d s \underset{t \rightarrow+\infty}{\longrightarrow} 0 .
$$

Ceci implique: $\forall v, w \in X_{f}$ tels que $w \in W^{s s}(v), \operatorname{Pf}(v)=\operatorname{Pf}(w)$.

Toute fonction $f \in L^{2}$ peut être approchée par une suite de fonctions lipschitziennes $f_{n}$ en norme $L^{2}$. Par continuité, $P f_{n}$ converge en norme $L^{2}$ vers $P f$. Quitte à passer à une sous-suite, on peut supposer que cette convergence 
a lieu sur un ensemble $X_{0}$ de mesure totale. Posons $X^{\prime}=X_{0} \cap \bigcap_{n} X_{f_{n}}$. Si $f$ est $g_{t}$-invariante, on obtient, pour tout $v, w \in X^{\prime}$ :

$$
\forall w \in W^{s s}(v), \quad f(v)=\operatorname{Pf}(v)=\lim P f_{n}(v)=\lim P f_{n}(w)=P f(w)=f(w) .
$$

On définit alors

$$
\widetilde{f}(v)= \begin{cases}0 & \text { si } W^{s s}(v) \cap X^{\prime}=\varnothing, \\ f(w) & \text { avec } w \in W^{s s}(v) \cap X^{\prime} \text { sinon. }\end{cases}
$$

Cette fonction est $W^{s s}$-invariante et coïncide avec $f$ sur $X^{\prime}$. Ceci termine la preuve.

EXERCICE. Soit $A \subset X$ un ensemble mesurable invariant par le flot. Montrez que $A$ coïncide, à un ensemble de mesure nulle près, avec un ensemble saturé par le feuilletage stable: $\exists I \subset X$ tel que $\mu\left(A \triangle \bigcup_{v \in I} W^{s s}(v)\right)=0$. A-t-on pour autant $\mu\left(\bigcup_{v \in A} W^{s s}(v) \backslash A\right)=0$ ?

L'argument précédent admet des généralisations lorsque la mesure est infinie. On renvoie à [C07] pour plus de détails. Démontrons maintenant un lemme qui va s'avérer crucial pour la suite.

LEMME. Soient $(X, \mathcal{T}, \mu),(Y, \mathcal{S}, \nu)$ deux espaces probabilisés et soit $f: X \times Y \rightarrow \mathbf{R}$ une fonction $L^{2}$. On suppose qu'il existe $\varphi_{1}: X \rightarrow \mathbf{R}$ et $\varphi_{2}: Y \rightarrow \mathbf{R}$ deux fonctions mesurables, $Z \subset X \times Y$ un sous-ensemble de $\mu \otimes \nu$-mesure totale, tels que:

$$
\forall(x, y) \in Z, \quad f(x, y)=\varphi_{1}(x), \quad f(x, y)=\varphi_{2}(y) .
$$

Alors $f$ est constante presque partout.

Preuve. D'après le théorème de Fubini, il existe $Y_{0} \subset Y$ de mesure totale et $x_{0} \in X$ tels que $\left\{x_{0}\right\} \times Y_{0} \subset Z$. Pour tout $(x, y) \in Z \cap\left(X \times Y_{0}\right)$, le point $\left(x_{0}, y\right)$ est dans $Z$, ce qui implique: $\varphi_{1}\left(x_{0}\right)=\varphi_{2}(y)=f(x, y)$. Le lemme est démontré.

Considérons maintenant un flot sur un espace métrique, préservant une mesure de probabilité, tel que les feuilletages stables et instables forment localement un système de coordonnées dans lequel la mesure est équivalente à une mesure produit. Formalisons cela par une définition ad hoc. 
DÉFINITION. Soit $X$ un espace métrique, $g_{t}: X \rightarrow X$ un flot borélien qui préserve une mesure de probabilité $\mu$ borélienne. On dira que $\mu$ est absolument continue relativement aux feuilletages stables et instables si, pour tout $v \in X$, on peut trouver un ouvert $U$ contenant $v$, un $\delta>0$ et un homéomorphisme $\phi: \mathbf{R}^{k} \times \mathbf{R}^{l} \rightarrow U$ tels que, pour tout $(x, y) \in \mathbf{R}^{k} \times \mathbf{R}^{l}$,

- $\phi\left(\{x\} \times \mathbf{R}^{l}\right) \subset W^{s s}(\phi(x, y))$,

- $\phi\left(\mathbf{R}^{k} \times\{y\}\right) \subset g_{]-\delta, \delta[} W^{s u}(\phi(x, y))$,

- $\phi^{*}\left(\mu_{\mid U}\right)$ est équivalente à une mesure produit $\mu_{1} \otimes \mu_{2}$.

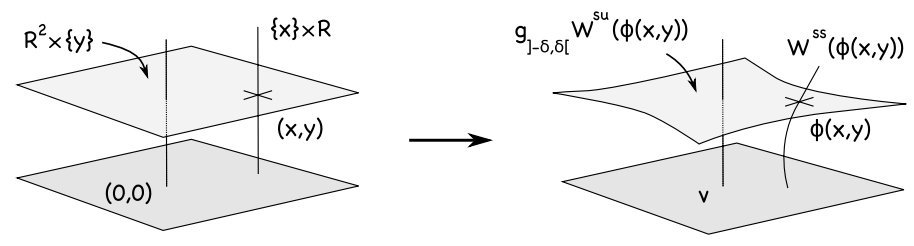

Supposons cette définition satisfaite, et considérons une fonction invariante par le flot. D'après l'argument de Hopf, elle est $W^{s s}$-invariante. Elle coïncide donc presque partout, dans la carte donnée par $\phi$, avec une fonction qui ne dépend que de la première coordonnée. En renversant la direction du flot, elle coïncide également avec une fonction qui ne dépend que de la seconde coordonnée. Par le lemme, elle est (presque) localement constante, et si le support de la mesure est connexe, on obtient l'ergodicité du flot.

THÉORÈME 4.1. Soit $X$ un espace métrique connexe, soit $g_{t}: X \rightarrow X$ un flot qui préserve une mesure de probabilité de support total, absolument continue par rapport aux feuilletages stables et instables. Alors le flot est ergodique relativement à cette mesure.

Revenons au cas du flot géodésique défini sur une variété à courbure strictement négative. Si la courbure est constante, l'absolue continuité se vérifie immédiatement dans un modèle algébrique pour le flot. C'est l'argument original de E. Hopf, qui établit par ce biais l'ergodicité du flot géodésique relativement au volume, dans un article fameux datant de 1936 [Ho36].

Si les feuilletages stables et instables sont $C^{1}$, l'absolue continuité est une conséquence du théorème du changement de variables. E. Hopf montre que les feuilletages sur les surfaces compactes à courbure négative pincée sont bien $C^{1}$ et en déduit l'ergodicité du volume en dimension deux. 
La question de l'absolue continuité en dimension supérieure reste ouverte jusqu'au début des années 60. D.V. Anosov résout finalement la question pour une classe générale de systèmes qui porte maintenant son nom.

THÉORÈME 4.2 (D. V. Anosov [An67]). Soit $g_{t}: X \rightarrow X$ un flot d'Anosov $C^{2}$ défini sur une variété riemannienne compacte connexe. On suppose que $g_{t}$ préserve le volume. Alors le volume est absolument continu relativement aux feuilletages stables et instables du flot.

Corollaire 4.3 (D.V. Anosov [An67]). Le flot géodésique défini sur le fibré unitaire d'une variété riemannienne $C^{3}$ connexe compacte à courbure strictement négative est ergodique relativement au volume.

Ce résultat est encore vrai dans le cas non compact, en supposant la variété complète, le volume fini et les dérivées partielles premières des courbures sectionnelles bornées.

La démonstration de l'absolue continuité donnée par D.V. Anosov est assez technique et cinquante ans plus tard, il n'y a pas réellement de simplification dans la preuve. Pour ce qui est de la régularité des feuilletages stables et instables, Hirsch et Pugh montrent en 1975 qu'ils sont $C^{1}$ si les courbures sectionnelles sont comprises strictement entre -4 et -1 . Ils ne sont en général pas $C^{\infty}$, comme on l'a mentionné plus haut.

\subsection{COMPORTEMENT RELATIF AU VOLUME}

On considère le flot géodésique sur une variété de rang un compacte à courbure négative ou nulle, et on se demande s'il est ergodique relativement au volume. Même dans le cas des surfaces, la question est ouverte.

QUESTION. Soit $M$ une surface compacte connexe orientable à courbure négative ou nulle, de genre supérieur ou égal à deux. Le flot géodésique défini sur le fibré unitaire de $M$ est-il ergodique relativement au volume?

On va présenter les travaux de Y. Pesin sur cette question. L'approche consiste à généraliser autant que possible l'argument de Hopf. Un ingrédient clef dans la preuve d'Anosov de l'absolue continuité des feuilletages, est la contraction/dilatation uniforme le long des feuilles stables et instables. Afin de récupérer un peu d'uniformité, il va falloir étudier les exposants de Lyapounov du flot. 
DÉfinition. Soit $g_{t}: X \rightarrow X$ un flot $C^{1}$ défini sur une variété riemannienne compacte et préservant une mesure de probabilité $\mu$ borélienne. Soit $v \in X, \xi \in T_{v} X$. Posons :

$$
\chi(v, \xi)=\lim _{t \rightarrow+\infty} \frac{1}{t} \ln \left\|d_{v} g_{t}(\xi)\right\| .
$$

$\mathrm{V}$. Oseledets [068] montre que cette limite existe pour $\mu$-presque tout $v \in X$ et pour tout $\xi \in T_{v} X$. On parle d'exposants associés au flot, et on dit que le flot n'a pas d'exposants nuls en $v$ (hormis dans la direction du flot), si $\operatorname{dim}\left\{\xi \in T_{v} X \mid \chi(v, \xi)=0\right\}=1$.

Les exposants ne sont pas définis partout en général.

QUESTION. Considérons le cas d'un flot géodésique sur une variété compacte à courbure négative ou nulle. Supposons que la limite définissant $\chi(v, \xi)$ existe en tout point $v \in X$. Peut-on en déduire que $M$ est localement symétrique?

Illustrons le concept d'exposants avec un point $v$ périodique de période $l$ sur le fibré unitaire $X$ d'une surface de rang un. Supposons par exemple que la géodésique engendrée par ce vecteur est dans un cylindre à courbure constante $K$ plongé dans la surface. Un calcul explicite donne:

$$
d_{v} g_{l}=\left(\begin{array}{ccc}
e^{\sqrt{|K|} l} & 0 & 0 \\
0 & 1 & 0 \\
0 & 0 & e^{-\sqrt{|K|} l}
\end{array}\right) \text {. }
$$

On obtient $\sqrt{|K|} l,-\sqrt{|K|} l$ et 1 comme valeurs possibles pour $\chi(v, \xi)$, quand $\xi$ varie dans $T_{v} X$. Les exposants sont non nuls (hormis dans la direction du flot) si la géodésique engendrée par $v$ est hyperbolique. Ils sont tous nuls si la géodésique périodique est dans un cylindre euclidien.

On veut maintenant travailler sur un ensemble où tous les exposants du flot sont non nuls (hormis dans la direction du flot).

THÉORÈME 4.4 (Y. Pesin [P77b]). Soit M une surface orientable connexe compacte à courbure négative ou nulle, de genre strictement supérieur à un. Notons $g_{t}$ le flot géodésique défini sur le fibré unitaire $X$ de cette surface et posons

$$
\Delta=\left\{v \in X \mid \varlimsup_{t \rightarrow+\infty} \frac{1}{t} \int_{0}^{t} K\left(g_{s}(v)\right) d s<0\right\} .
$$


Alors :

- l'ensemble $\Delta$ est invariant par le flot géodésique,

- le volume de $\Delta$ est non $\mathrm{nul}$,

- l'ensemble $\Delta$ est dense et ouvert $(\bmod 0)$,

- $\forall v \in \Delta$, les exposants sont non nuls (hormis dans la direction du flot),

- le flot est ergodique en restriction à $\Delta$, relativement au volume.

Expliquons brièvement comment se démontre le théorème. Seul le dernier point est propre à la mesure de Lebesgue. Les autres points sont satisfaits relativement à toute mesure $\mu$ invariante de support total.

- Le premier point est clair.

- Le second découle du théorème ergodique; si $\Delta$ est de mesure nulle, la limite dans le théorème ergodique doit être positive presque partout. $\mathrm{Ce}$ n'est pas possible car son intégrale est égale à $\int_{M} K d \mu<0$.

- Comme les orbites périodiques hyperboliques sont denses dans $M$ (cf.plus haut) et contenues dans $\Delta$, l'ensemble $\Delta$ est bien dense dans $X$. Vérifions que $\Delta$ est (presque) ouvert. Tout vecteur $v$ dans $\Delta$ admet un voisinage dont l'image par $g_{T}$, pour un certain $T>0$, est contenu dans une boîte de flot sur laquelle la courbure est majorée par $-\epsilon$, pour un certain $\epsilon>0$. D'après le théorème ergodique de Birkhoff appliqué à $g_{1}$, presque tous les points de la boîte reviennent dans la boîte avec une fréquence strictement positive. Ces points sont dans $\Delta$.

- Le lien entre courbure et exposants se fait par le biais des champs de Jacobi. Dans un système de coordonnées bien choisi, la différentielle du flot se met sous la forme $d_{v} g_{t}=\left(J(t), J^{\prime}(t)\right)$, où $J$ est un champ de vecteurs le long de la géodésique engendré par $v$ qui satisfait $J^{\prime \prime}(t)=-K\left(g_{t} v\right) J(t)$. De là on vérifie que les exposants sont non nuls sur $\Delta$.

Pour démontrer l'ergodicité, on fait appel à la théorie des systèmes non uniformément hyperboliques.

THÉORÈME 4.5 (Y. Pesin [P77a]). Soit $X$ une variété riemannienne compacte, $g_{t}: X \rightarrow X$ un flot $C^{1}$ qui préserve le volume. Notons $\Lambda$ l'ensemble des points sans exposants nuls (hormis dans la direction du flot). On suppose $\operatorname{vol}(\Lambda)>0$. Alors, on peut trouver des ensembles $\Lambda_{i}$, en nombre au plus dénombrable, disjoints deux à deux, de volume non nul, dont l'union est égale à $\Lambda$ à un ensemble négligeable près, et tels que $g_{t}$ est ergodique relativement au volume en restriction à chacun des $\Lambda_{i}$. 
Donnons un bref aperçu de la preuve. Il s'agit de récupérer de la contraction/dilatation uniforme le long des feuilles stables afin de montrer l'absolue continuité. Posons :

$$
W_{\text {exp }}^{s s}(v)=\left\{w \in X \mid \varlimsup_{t \rightarrow+\infty} \frac{1}{t} \ln d\left(g_{t}(v), g_{t}(w)\right)<0\right\} .
$$

Grâce à la non nullité des exposants, on montre que pour presque tout point de $\Lambda$, ces ensembles forment des courbes $C^{1}$ immergées. Ces courbes ne varient pas continûment en fonction du point. En utilisant le théorème d'Egorov, on peut tout de même obtenir des sous-ensembles de mesure positive sur lesquels on récupère la continuité et une contraction uniforme sur les $W_{\text {exp }}^{s s}(v)$. Ces sous-ensembles ne sont pas invariants; on fait malgré tout fonctionner la preuve de l'absolue continuité pour ces feuilletages et un passage à la limite donne un ensemble invariant de mesure positive sur lequel le flot est ergodique. L'ensemble $\Lambda$ est de mesure finie, il ne peut donc contenir qu'un nombre dénombrable d'ensembles disjoints de mesure strictement positive.

Revenons au flot géodésique. Pour obtenir l'ergodicité en restriction à $\Delta$, il faut démontrer que les ensembles $\Lambda_{i}$ sont ouverts (mod 0). L'ingrédient crucial dans cette dernière étape de la preuve est l'inclusion:

$$
W_{e x p}^{s s}(v) \subset W^{s s}(v) \subset \operatorname{Hor}^{+}(v) .
$$

Comme les horosphères varient continûment en fonction du point, on récupère un peu de régularité sur les $W_{\text {exp }}^{s s}$, ce que la théorie générale ne fournissait pas. De là, on montre que les $\Lambda_{i}$ sont des sous-ensembles ouverts $(\bmod 0)$ et on conclut par transitivité. En corollaire, on obtient l'égalité $W_{\text {exp }}^{s s}(v)=\operatorname{Hor}^{+}(v)$ pour presque tout $v \in \Delta$. On pourra consulter [BP02] pour plus de détails.

Remarquons pour finir que si l'ensemble des vecteurs de rang un est de volume total, alors pour presque tout $v \in T^{1} M$, on peut trouver $t \in \mathbf{R}$ tel que $K\left(g_{t}(v)\right)<0$. Le lemme suivant, qui découle du théorème ergodique de Birkhoff, montre alors que l'ensemble $\Delta$ est de volume total.

LEMME. Soit $X$ un espace métrique, $g_{t}: X \rightarrow X$ un flot continu qui préserve une mesure de probabilité $\mu$, et $f: X \rightarrow \mathbf{R}_{+}$une fonction continue intégrable positive. Alors, pour presque tout $x \in X$, l'existence d'un $t \in \mathbf{R}$ tel que $f\left(g_{t} x\right)>0$ implique l'existence d'une limite strictement positive pour $\frac{1}{T} \int_{0}^{T} f\left(g_{t} x\right) d t$ quand $T \rightarrow \infty$. 
L'ergodicité du volume se ramène donc à la question suivante:

QUESTION. Soit $S$ une surface connexe orientable compacte de rang un. L'ensemble des vecteurs de rang deux est-il de volume nul?

Cette question admet une réponse positive lorsque la surface et sa métrique sont réelles-analytiques, ou encore lorsque l'ensemble des points de courbure strictement négative possède un nombre fini de composantes connexes [BP06, $\S 17]$.

\subsection{LA MESURE D'ENTROPIE MAXIMALE}

LE CAS DE LA COURBURE STRICTEMENT NÉGATIVE. Le volume n'est pas la seule mesure invariante par le flot géodésique qui présente un intérêt. A partir des années soixante, une autre mesure va jouer un rôle important dans la description de la distribution des géodésiques périodiques. Il s'agit de la mesure de Bowen-Margulis.

La construction de Margulis [M70] passe par les feuilletages stables et instables. Soit $g_{t}: X \rightarrow X$ un flot d'Anosov $C^{1}$ transitif défini sur une variété compacte connexe. G. Margulis montre qu'il existe une famille de mesures $\mu_{W^{s s}(x)}$ supportées par les feuilles stables fortes, qui est contractée exponentiellement par le flot:

$$
g_{t_{*}} \mu_{W^{s s}(x)}=e^{-h t} \mu_{W^{s s}\left(g_{t}(x)\right)} .
$$

La difficulté ici est de définir précisément ce qu'est l'espace des mesures portées par les feuilles, l'existence et l'unicité découlent du théorème du point fixe contractant. De la même façon, il existe une famille de mesures $\mu_{W^{u}}(x)$ portées par les feuilles instables faibles $g_{\mathbf{R}} W^{s u}(x)$ qui est dilatée par le flot.

On peut alors faire le produit de ces deux mesures dans un système de coordonnées données par les feuilles stables et instables. La mesure obtenue est la mesure de Margulis. Comme elle est par construction absolument continue relativement aux feuilletages stables et instables, son ergodicité découle de l'argument de Hopf.

L'approche de Bowen est de nature différente. Il considère des moyennes de mesures de Dirac sur les orbites périodiques de période inférieure à un certain réel $T$ donné, et montre que ces moyennes convergent lorsque $T$ tend vers l'infini. Notons $\mathcal{P}$ l'ensemble des orbites périodiques du flot, et $l(c)$ la période d'une orbite périodique $c$ dans $X$. 
THÉORÈME 4.6 (R. Bowen [B72], [B74]). Soit $g_{t}$ un flot d'Anosov transitif défini sur une variété compacte connexe. Alors il existe une mesure $\mu$ telle que:

$$
\frac{1}{\#\{c \in \mathcal{P} \mid l(c) \leq t\}} \sum_{c \in \mathcal{P} \text { tq } l(c) \leq t} \delta_{c} \underset{t \rightarrow \infty}{\longrightarrow} \mu .
$$

L'entropie de cette mesure est égale à l'entropie topologique du flot, et toutes les autres mesures de probabilités invariantes ont une entropie strictement inférieure à celle de $\mu$.

La preuve consiste à calculer explicitement l'entropie des points d'accumulation des moyennes qui viennent d'être décrites. Ce calcul est basé sur deux propriétés du flot connues sous le nom d'expansivité et de spécification. La propriété de spécification est en fait suffisante pour montrer qu'il existe au plus une mesure dont l'entropie coïncide avec l'entropie topologique. Il n'y a donc qu'un seul point d'accumulation possible et les moyennes sont convergentes. L'ergodicité de la mesure d'entropie maximale est une conséquence immédiate de son unicité et de la convexité de l'entropie.

Il faut mentionner que la mesure d'entropie maximale est tout d'abord construite par W. Parry [P64] dans le contexte des décalages sur les chaînes de Markov. A cette date, on connaît un certain nombre de variétés à courbure négative sur lesquelles le flot géodésique est conjugué à une suspension d'une chaîne de Markov; c'est le cas par exemple pour les surfaces compactes à courbure constante négative. L'existence et l'unicité de la mesure d'entropie maximale est donc connue dès 1964 pour ces variétés.

LE CAS DES VARIÉTÉS DE RANG UN. G. Knieper [Kn98] parvient en 1998 à généraliser le théorème de Bowen aux flots géodésiques définis sur les variétés $M$ compactes de rang un. Les moyennes doivent porter sur les géodésiques périodiques hyperboliques; on a alors convergence de ces moyennes vers l'unique mesure d'entropie maximale. Présentons brièvement les travaux de G. Knieper.

La construction de la mesure d'entropie maximale passe par le bord du revêtement universel, suivant une idée due à S. Patterson [P76]. Rappelons comment est défini le bord idéal de $\widetilde{M}$. On introduit une relation d'équivalence sur l'ensemble des demi-géodésiques $c: \mathbf{R}_{+} \rightarrow \widetilde{M}$ comme suit: deux demigéodésiques sont dites asymptotiques si elles sont à distance bornée l'une de l'autre pour tout temps positif. Le bord $\partial \widetilde{M}$ est l'ensemble des classes d'équivalence associées à cette relation. Dans le cas du disque de Poincaré, ce bord idéal s'identifie au cercle unité. Paramétrer les géodésiques par le biais 
de leurs extrémités sur le bord du disque est une idée qui remonte au début du vingtième siècle; on parle parfois de coordonnées de Hopf.

Notons $\Gamma$ le groupe du revêtement $\widetilde{M} \rightarrow M$. Il se plonge naturellement dans le groupe d'isométries de $\widetilde{M}$ et $M$ s'identifie au quotient $\widetilde{M} / \Gamma$. Fixons un point $p \in \widetilde{M}$ et notons $h_{\text {top }}$ l'entropie topologique du flot. La mesure sur le bord est construite à partir de limites faibles de la forme:

$$
\frac{\sum_{\gamma \in \Gamma} e^{-s d(p, \gamma(p))} \delta_{\gamma(p)}}{\sum_{\gamma \in \Gamma} e^{-s d(p, \gamma(p))}}
$$

G. Knieper montre que la série au dénominateur est convergente pour $s<h_{t o p}$ et diverge pour $s \geq h_{\text {top }}$. Lorsque $s$ converge vers $h_{\text {top }}$, les moyennes convergent vers une mesure $\mu_{p}$ portée par le bord, qui est de support total.

Il s'agit maintenant de récupérer une mesure sur $T^{1} M$. Pour cela, on considère la projection $P: T^{1} M \rightarrow \partial \widetilde{M} \times \partial \widetilde{M}$ qui associe à un vecteur les deux extrémités de la géodésique qu'il engendre. Seuls les couples de points du bord qui peuvent être effectivement joints par une géodésique sont dans l'image de $P$. Les lignes de niveau de $P$ sont constituées de géodésiques ou de plats; on munit ces ensembles de la mesure de Lebesgue $\lambda$. On définit alors une mesure $\mu$ sur $T^{1} M$ en posant:

$$
\mu(A)=\int_{P\left(T^{1} M\right)} \lambda\left(\left\{P^{-1}(\xi, \eta) \cap A\right\}\right) h(\xi, \eta) d \mu_{p}(\xi) d \mu_{p}(\eta)
$$

avec $h$ une densité bien choisie de manière à obtenir une mesure invariante par le flot. L'ergodicité de $\mu$ s'obtient à l'aide de l'argument de Hopf et du lemme élémentaire suivant:

LeMme 4.7. Soit $v$ un vecteur de $T^{1} M$ récurrent de rang un. Alors $W^{s s}(v)=\operatorname{Hor}^{+}(v)$.

Preuve. On travaille dans $T^{1} \widetilde{M}$ et on note encore $v$ un relevé de $v$ à $T^{1} \widetilde{M}$. Considérons un point $w \in \operatorname{Hor}^{+}(v)$ qui n'appartient pas à $W^{s s}(v)$. On peut trouver $C>0$ tel que:

$$
\forall t \geq 0, \quad C \leq d\left(g_{t}(v), g_{t}(w)\right) \leq d(v, w) .
$$

Par récurrence, il existe $\gamma_{n} \in \Gamma, t_{n} \rightarrow+\infty$ tels que $\gamma_{n}\left(g_{t_{n}}(v)\right) \rightarrow v$. On a donc pour tout $s \geq-t_{n}$,

$$
C \leq d\left(g_{t_{n}+s}(v), g_{t_{n}+s}(w)\right)=d\left(g_{s} \gamma_{n} g_{t_{n}}(v), g_{s} \gamma_{n} g_{t_{n}}(w)\right) \leq d(v, w) .
$$

Quitte à passer à une sous-suite, on peut supposer que la suite $\gamma_{n} g_{t_{n}}(w)$ 
converge vers une limite $w^{\prime}$. On a alors, pour tout $s \in \mathbf{R}$,

$$
C \leq d\left(g_{s}(v), g_{s}\left(w^{\prime}\right)\right) \leq d(v, w) .
$$

Le vecteur $v$ est sur le bord d'une bande plate, il est donc de rang plus grand que un. Ceci termine la preuve.

Le calcul de l'entropie de $\mu$ s'inspire de la méthode mise au point par R. Bowen. Le flot géodésique sur une variété de rang un n'est pas expansif en général, mais il satisfait une propriété plus faible appelée $h$-expansivité. De même, le flot ne possède plus la propriété de spécification, mais on a tout de même un produit local en restriction aux vecteurs de rang un. Il devient alors possible de montrer que les mesures invariantes singulières par rapport à $\mu$ ont une entropie plus petite que celle de $\mu$. On renvoie à [B74] et [Kn02] pour un aperçu des calculs.

QUESTION. Est-il possible de généraliser les considérations précédentes au cas des variétés non compactes?

VOLUME ET MESURE D'ENTROPIE MAXIMALE. En courbure négative constante, la mesure d'entropie maximale coïncide avec le volume riemannien. En courbure négative variable, par contre, elles sont en général distinctes:

THÉORÈME 4.8 (A. Katok [K82]). Soit $S$ une surface compacte connexe à courbure strictement négative. La mesure d'entropie maximale et le volume riemannien coïncident si et seulement si la courbure est constante.

La question est ouverte en dimension supérieure.

\subsection{LE POINT DE VUE GÉNÉRIQUE}

On peut se demander si l'ergodicité est une propriété typique des mesures de probabilités invariantes par le flot géodésique.

En courbure strictement négative, K. Sigmund étudie la question sous l'angle de la généricité. Rappelons qu'un sous-ensemble d'un espace métrique est un $G_{\delta}$-dense si c'est une intersection dénombrable d'ouverts denses. Si le théorème de Baire est valide dans $X$, alors un tel ensemble est lui-même dense; en particulier, il est non vide. Remarquons qu'une intersection dénombrable de $G_{\delta}$-denses est encore un $G_{\delta}$-dense. Pour cette raison, ces ensembles sont parfois considérés comme un analogue topologique des ensembles de mesure totale en théorie de la mesure. 
On dit qu'une propriété associée à certains points d'un espace métrique $X$ est générique si l'ensemble des points qui satisfont cette propriété contient un sous-ensemble $G_{\delta}$-dense.

Le flot géodésique en courbure négative possède de nombreuses mesures de probabilités ergodiques, par exemple tous les Diracs portés par des orbites périodiques. On construit également facilement des mesures de support total. On peut par exemple considérer une somme de tels Diracs: $\sum 2^{-i} \delta_{c_{i}}$; dès que les orbites périodiques $c_{i}$ forment un ensemble dense dans $X$, la mesure obtenue est de support total (mais pas ergodique).

La complexité inhérente à la preuve de l'ergodicité du volume ou à la construction de la mesure d'entropie maximale pourrait laisser penser que peu de mesures invariantes sont ergodiques.

ThÉORÈme 4.9 (K. Sigmund [Si72]). Soit $g_{t}: X \rightarrow X$ un flot d'Anosov transitif défini sur une variété compacte $C^{1}$. Alors l'ensemble des mesures invariantes ergodiques de support total est un $G_{\delta}$-dense dans l'ensemble de toutes les mesures de probabilités boréliennes invariantes définies sur $X$.

Ici, on a muni l'ensemble $\mathcal{M}^{1}(X)$ des mesures de probabilités boréliennes invariantes sur $X$ de la topologie associée à la convergence étroite: une suite de mesures de probabilités $\mu_{n}$ convergent vers $\mu$ si pour toute fonction $f$ continue bornée, $\int f d \mu_{n}$ converge vers $\int f d \mu$. Quand $X$ est un espace métrique séparable complet, l'espace $\mathcal{M}^{1}(X)$ est lui aussi métrisable et complet pour une certaine distance qui redonne la topologie.

Rappelons que les mesures de probabilités invariantes sont toutes supportées par l'ensemble non errant du flot. Si on veut un énoncé valide dans le cas non compact, il faut donc se restreindre à l'ensemble des mesures de probabilités supportées par cet ensemble.

Remarquons de plus que l'existence d'une mesure de probabilité invariante ergodique de support total dans $\Omega$ implique la transitivité du flot en restriction à $\Omega$. On a vu plus haut un exemple de surface de rang un non compacte sur laquelle cette propriété n'était pas satisfaite.

THÉORÈmE 4.10 (Y. Coudène, B. Schapira [CS10]). Soit M une variété de rang un telle que tous les vecteurs de $T^{1} M$ sont non errants sous l'action du flot géodésique: $\Omega=T^{1} M$. Soit $\mathcal{R}_{1} \subset T^{1} M$ l'ouvert des vecteurs de rang un. Alors l'ensemble des mesures définies sur $\mathcal{R}_{1}$ qui sont invariantes par le flot, ergodiques, de support total dans $\mathcal{R}_{1}$, est un $G_{\delta}$-dense dans l'ensemble de toutes les mesures de probabilités invariantes définies sur $\mathcal{R}_{1}$. 
Sous les hypothèses du théorème, l'ouvert $\mathcal{R}_{1}$ est dense dans $T^{1} M$, si bien que les mesures données par le théorème sont aussi des mesures ergodiques invariantes de support total dans $T^{1} M$. De plus l'ouvert $\mathcal{R}_{1}$ est non vide invariant, si bien que toute mesure ergodique de support total dans $T^{1} M$ est supportée par $\mathcal{R}_{1}$.

Même en courbure constante -1 , l'existence d'une mesure de probabilité ergodique de support total est non triviale. En général, sur les variétés non compactes, le volume est totalement dissipatif et "la" mesure de BowenMargulis n'est pas définie.

COROllaire 4.11. Soit $M$ une variété de rang un telle que $\Omega=T^{1} M$. Alors il existe une mesure de probabilité invariante par le flot géodésique, ergodique et de support total.

Cependant, les mesures ergodiques de support total ne forment pas toujours un sous-ensemble dense dans l'ensemble de toutes les mesures de probabilités invariantes.

PROPOSITION 4.12. Soit $M$ une surface compacte admettant un cylindre euclidien plongé. Alors les Diracs supportés par les orbites périodiques à l'intérieur du cylindre ne sont pas dans l'adhérence des mesures de probabilités invariantes ergodiques de support total.

Preuve. Soit $c: \mathbf{R} \rightarrow M$ une géodésique périodique à l'intérieur du cylindre. Considérons un voisinage tubulaire de $c$ dans le cylindre isométrique à $]-3 \varepsilon, 3 \varepsilon\left[\times S^{1}\right.$.

Soit $\theta \in] 0, \frac{\pi}{2}\left[\right.$. Considérons l'ouvert $U_{\varepsilon}$ constitué de tous les vecteurs tangents à $]-\varepsilon, \varepsilon\left[\times S^{1}\right.$ et faisant un angle avec la verticale compris dans l'intervalle $]-\theta, \theta[$.

Les orbites du flot géodésique sont des droites dans le cylindre, si bien qu'une trajectoire qui rentre dans $U_{\varepsilon}$ au temps $t_{0}$ et qui en sort au temps $t_{1}$, a dû passer d'abord un temps au moins égal à $t_{1}-t_{0}$ dans un des cylindres $]-3 \varepsilon,-\varepsilon] \times S^{1}$ ou $\left[\varepsilon, 3 \varepsilon\left[\times S^{1}\right.\right.$. Par conséquent, la quantité : $\frac{1}{T} \lambda\left\{t \in[0, T] \mid g_{t}(v) \in U_{\varepsilon}\right\}$ est inférieure ou égale à $\frac{1}{2}$ pour tout $T>0$ et tout $v \in T^{1} M$ extérieur au cylindre.

Soit $\mu$ une mesure ergodique de support total; si elle est suffisamment proche du Dirac porté par la géodésique $c$, on a: $\mu\left(U_{\varepsilon}\right)>\frac{1}{2}$. Appliquons le théorème ergodique. On peut trouver une orbite $v \in T^{1} M$ hors du cylindre 
telle que:

$$
\frac{1}{T} \lambda\left(\left\{t \in[0, T] \mid g_{t}(v) \in U_{\varepsilon}\right\}\right) \underset{T \rightarrow \infty}{\longrightarrow} \mu\left(U_{\varepsilon}\right),
$$

ce qui donne une contradiction. La proposition est démontrée.

QUESTION. Peut-on caractériser les variétés compactes de rang un pour lesquelles les mesures ergodiques de support total forment un $G_{\delta}$-dense dans l'ensemble de toutes les mesures de probabilité invariantes?

Dans le cas où il existe des vecteurs errants, on peut quand même démontrer la généricité des mesures ergodiques de support total, si on se restreint à l'ensemble $\mathcal{R}_{1} \cap \Omega$ des vecteurs de rang un non errants. Ceci implique en particulier:

THÉORÈME 4.13 (Y. Coudène, B. Schapira [CS10]). Soit $M$ une variété connexe complète à courbure négative ou nulle. On suppose que l'ensemble non errant du flot géodésique contient plus de deux orbites périodiques hyperboliques. Alors il existe une mesure de probabilité invariante ergodique dont le support contient toutes les géodésiques périodiques hyperboliques.

Expliquons brièvement comment se démontrent ces théorèmes. La preuve de K. Sigmund est basée sur la propriété de spécification. Celle-ci ne peut être satisfaite que si l'espace ambiant est compact, et semble difficile à obtenir en l'absence d'hyperbolicité uniforme.

Nous sommes parvenu à montrer la densité des mesures ergodiques de support total en nous basant sur deux propriétés du flot à priori plus faibles que la spécification: l'existence d'une structure de produit local et le lemme de fermeture. Ces propriétés ne nécessitent à priori pas d'hypothèse de compacité sur l'espace ambiant, et ne reposent que sur une forme faible d'hyperbolicité. Il a été possible de les établir dans le contexte des variétés de rang un.

Pour cela, il faut se restreindre à un sous-ensemble de la variété sur lequel on observe les phénomènes de dilatation/contraction propres à la courbure strictement négative. Nous nous sommes placés sur l'ensemble $\Omega_{1}$ des vecteurs de rang un non errants, pour lesquels variétés stables et horosphères stables coïncident. On récupère sur cet ensemble la structure de produit local et le lemme de fermeture.

Cet ensemble $\Omega_{1}$ contient les vecteurs de rang un récurrents, d'après le lemme de G. Knieper mentionné plus haut. Il est donc de mesure totale pour toute mesure de probabilité invariante portée par les vecteurs de rang un, 
d'après le théorème de récurrence de Poincaré. Ce qui se passe hors de $\Omega_{1}$ est invisible du point de vue des mesures invariantes, et le flot se comporte de manière suffisamment proche d'un flot hyperbolique sur $\Omega_{1}$, pour qu'on puisse obtenir la généricité en restriction à $\Omega_{1}$. Enfin, le lemme de fermeture montre que les géodésiques périodiques de rang un sont denses dans l'ensemble des vecteurs de rang un non errants, ce qui permet de conclure à la densité de $\Omega_{1}$ dans $T^{1} M$ si $\Omega=T^{1} M$.

\section{BIBLIOGRAPHIE}

[An67] ANOSOV, D.V. Geodesic flows on closed Riemannian manifolds with negative curvature. Proc. Steklov Inst. Math 90 (1967), 209 pp.

[B85] BaLlmann, W. Nonpositively curved manifolds of higher rank. Ann. of Math. (2) 122 (1985), 597-609.

[B95] Lectures on Spaces of Nonpositive Curvature. With an appendix by M. Brin. DMV Seminar 25. Birkhäuser Verlag, Basel, 1995.

[BBB87] BALlMANN, W., M. BRIN and K. BuRns. On the differentiability of horocycles and horocycle foliations. J. Differential Geom. 26 (1987), 337-347.

[BBE85] BALlMANN, W., M. BRIN and P. EBERLEIN. Structure of manifolds of nonpositive curvature. I. Ann. of Math. (2) 122 (1985), 171-203.

[BGS85] Ballmann, W., M. Gromov and V. Schroeder. Manifolds of Nonpositive Curvature. Progress in Mathematics 61. Birkhäuser Boston, Inc., Boston, MA, 1985.

[BP02] BARREIRA, L. and Y.B. PESIN. Lyapunov Exponents and Smooth Ergodic Theory. University Lecture Series 23. Amer. Math. Soc., Providence, RI, 2002.

[BP06] BARREIRA, L. and Y.B. PESIN. Smooth ergodic theory and nonuniformly hyperbolic dynamics. With an appendix by O.Sarig. In: Handbook of Dynamical Systems. Vol.1B, 57-263. Elsevier B. V., Amsterdam, 2006.

[BCG95] Besson, G., G. Courtols et S. Gallot. Entropies et rigidités des espaces localement symétriques de courbure strictement négative. Geom. Funct. Anal. 5 (1995), 731-799.

[B72] Bowen, R. Periodic orbits for hyperbolic flows. Amer. J. Math. 94 (1972), 1-30.

[B74] Maximizing entropy for a hyperbolic flow. Math. Systems Theory 7 (1974), 300-303. 
[C05] CoudÈnE, Y. A note on horospherical points for flows with hyperbolic structure. Ergodic Theory Dynam. Systems 25 (2005), 793-798.

[C07] - The Hopf Argument. J. Modern Dynamics 1 (2007), 147-153.

[CS10] COUdÈnE, Y. and B. SCHAPIRA. Generic measures for hyperbolic flows on non-compact spaces. Israel J. Math. 179 (2010), 157-172.

[D00] DAL'BO, F. Topologie du feuilletage fortement stable. Ann. Inst. Fourier (Grenoble) 50 (2000), 981-993.

[Eb96] EberLein, P.B. Geometry of Nonpositively Curved Manifolds. Chicago Lectures in Mathematics. University of Chicago Press, Chicago, IL, 1996.

[Eb72] Geodesic flows on negatively curved manifolds. I. Ann. of Math. (2) 95 (1972), 492-510.

[Eb73] - Geodesic flows on negatively curved manifolds. II. Trans. Amer. Math. Soc. 178 (1973), 57-82.

[H-IH77] HeInTZE, E. and H.-C. IM HoF. Geometry of horospheres. J. Differential Geom. 12 (1977), 481-491.

[Ho36] Hopf, E. Fuchsian groups and ergodic theory. Trans. Amer. Math. Soc. 39 (1936), 299-314.

[K82] KATOK, A. Entropy and closed geodesics. Ergodic Theory Dynam. Systems 2 (1982), 339-365.

[Kn98] KNIEPER, G. The uniqueness of the measure of maximal entropy for geodesic flows on rank 1 manifolds. Ann. of Math. (2) 148 (1998), 291-314.

[Kn02] - Hyperbolic dynamics and Riemannian geometry. In: Handbook of Dynamical Systems, Vol. 1A, 453-545, North-Holland, Amsterdam, 2002.

[LPP06] Link, G., M. PeignÉ et J.-C. PiCAUd. Sur les surfaces non-compactes de rang un. L'Enseignement Math. (2) 52 (2006), 3-36.

[M70] Margulis, G.A. Certain measures associated with U-flows on compact manifolds. Funct. Anal. Appl. 4 (1970), 55-67.

[O68] OSELEDETS, V.I. A multiplicative ergodic theorem. Liapunov characteristic numbers for dynamical systems. Trans. Moscow Math. Soc. 19 (1968), 197-221.

[P64] PARRY, W. Intrinsic Markov chains. Trans. Amer. Math. Soc. 112 (1964), $55-66$.

[P76] PATtERSON, S.J. The limit set of a Fuchsian group. Acta Math. 136 (1976), 241-273.

[P77a] PesIN, YA.B. Characteristic Lyapounov exponents, and smooth ergodic theory. Russian Math. Surveys 32 (1977), 55-114.

[P77b] Geodesic flows on closed Riemannian manifolds without focal points. Math. USSR Izv. 11 (1977), 1195-1228.

[S10] SCHAPIRA, B. Density and equidistribution of half horocycles on geometrically finite surfaces. J. London Math. Soc., to appear. 
[Si72] Sigmund, K. On the space of invariant measures for hyperbolic flows. Amer. J. Math. 94 (1972), 31-37.

(Reçu le 14 juillet 2010)

\section{Yves Coudène}

Département de mathématiques

Université de Bretagne Occidentale

6 avenue Le Gorgeu

29238 Brest cedex 3

France

e-mail: yves.coudene@univ-brest.fr 